Author Manuscript Published OnlineFirst on September 24, 2015; DOI: 10.1158/1055-9965.EPI-15-0422

Author manuscripts have been peer reviewed and accepted for publication but have not yet been edited.

\title{
The Association between Glyceraldehyde-derived Advanced Glycation End-Products and Colorectal Cancer Risk
}

So Yeon Kong ${ }^{1}$, Masayoshi Takeuchi ${ }^{2}$, Hideyuki Hyogo ${ }^{3}$, Gail McKeown-Eyssen ${ }^{4}$, Sho-ichi Yamagishi ${ }^{5}$, Kazuaki Chayama ${ }^{3}$, Peter J. O'Brien ${ }^{6}$, Pietro Ferrari ${ }^{1}$, Kim Overvad ${ }^{7}$, Anja Olsen ${ }^{8}$, Anne Tjønneland ${ }^{8}$, Marie-Christine Boutron-Ruault ${ }^{9}$, Nadia Bastide ${ }^{9}$, Franck Carbonnel ${ }^{9,10}$, Tilman Kühn ${ }^{11}$, Rudolf Kaaks ${ }^{11}$, Heiner Boeing ${ }^{12}$, Krasimira Aleksandrova ${ }^{12}$, Antonia Trichopoulou ${ }^{13,14,15}$, Pagona Lagiou ${ }^{14,15,16}$, Effie Vasilopoulou $^{14}$, Giovanna Masala ${ }^{17}$, Valeria Pala ${ }^{18}$, Maria Santucci De Magistris ${ }^{19}$, Rosario Tumino ${ }^{20}$, Alessio Naccarati ${ }^{21}$, H.B. Bueno-de-Mesquita ${ }^{22,23,24,25}$, Petra H. Peeters ${ }^{26,27}$, Elisabete Weiderpass ${ }^{28,29,30,31}$, J. Ramón Quirós ${ }^{32}$, Paula Jakszyn ${ }^{33}$, María-José Sánchez ${ }^{34,35}$, Miren Dorronsoro ${ }^{36}$, Diana Gavrila $^{34,37}$, Eva Ardanaz ${ }^{34,38}$, Martin Rutegård ${ }^{39}$, Hanna Nyström ${ }^{39}$, Nicholas J. Wareham ${ }^{40}$, KayTee Khaw ${ }^{41}$, Kathryn E. Bradbury ${ }^{42}$, Isabelle Romieu ${ }^{1}$, Heinz Freisling ${ }^{1}$, Faidra Stavropoulou ${ }^{1 *}$, Marc J Gunter $^{43}$, Amanda J Cross ${ }^{43}$, Elio Riboli ${ }^{43}$, Mazda Jenab $^{1 * *}$, W. Robert Bruce ${ }^{44 * *}$

${ }^{1}$ Section of Nutrition and Metabolism, International Agency for Research on Cancer (IARC-WHO), Lyon, France.

${ }^{2}$ Department of Advanced Medicine, Medical Research Institute, Kanazawa Medical University, Kanazawa, Japan

${ }^{3}$ Department of Gastroenterology and Metabolism, Hiroshima University Hospital, Hiroshima, Japan.

${ }^{4}$ Dalla Lana School of Public Health, University of Toronto, Toronto, Canada

${ }^{5}$ Department of Pathophysiology and Therapeutics of Diabetic Vascular Complications, Kurume University School of Medicine, Kurume, Japan

${ }^{6}$ Department of Pharmacology, University of Toronto, Toronto, Canada

${ }^{7}$ Section for Epidemiology, Department of Public Health, Aarhus University, Aarhus, Denmark

${ }^{8}$ Diet, Genes and Environment, Danish Cancer Society Research Center, Copenhagen, Denmark

${ }^{9}$ Lifestyle, Genes and Health: Trans-generational Integrated Epidemiology, EMT, Institute Gustave Roussy, Villejuif, France

${ }^{10}$ Service d'hépato-gastroentérologie, Hôpital Bicetre, 94275 Le Kremlin-Bicêtre Cedex, France

${ }^{11}$ Division of Cancer Epidemiology, German Cancer Research Center (DKFZ), Heidelberg, Germany

${ }^{12}$ Department of Epidemiology, German Institute of Human Nutrition Potsdam-Rehbrücke, Nuthetal, Germany

${ }^{13}$ Hellenic Health Foundation, Athens Greece

${ }^{14}$ Department of Hygiene, Epidemiology and Medical Statistics, University of Athens Medical School, Athens, Greece

${ }^{15}$ Bureau of Epidemiologic Research, Academy of Athens, Athens, Greece

${ }^{16}$ Department of Epidemiology, Harvard School of Public Health, Boston, USA

${ }^{17}$ Molecular and Nutritional Epidemiology Unit, Cancer Research and Prevention Institute - ISPO,

Florence, Italy

${ }^{18}$ Epidemiology and Prevention Unit, IRCCS Foundation, National Cancer Institute, Milan, Italy

${ }^{19}$ Azienda Ospedaliera Universitaria (AOU) Federico II, Naples, Italy

${ }^{20}$ Cancer Registry and Histopathology Unit, "Civic - MP Arezzo" Hospital, Ragusa, Italy

${ }^{21}$ Human Genetics Foundation, Torino Molecular and Genetic Epidemiology Unit, Torino, Italy

${ }^{22}$ Department for Determinants of Chronic Diseases DCD), National Institute for Public Health and Environment (RIVM), Bilthoven, The Netherlands

${ }^{23}$ Department of Gastroenterology and Hepatology, University Medical Centre, Utrecht, The Netherlands

${ }^{24}$ Department of Epidemiology and Biostatistics, The School of Public Health, Imperil College London, London, United Kingdom 
${ }^{25}$ Department of Social \& Preventive Medicine, Faculty of Medicine, University of Malaya, Kuala Lumpur, Malaysia

${ }^{26}$ Department of Epidemiology, Julius Center for Health Sciences and Primary Care, University Medical Center Utrecht, Utrecht, the Netherlands

${ }^{27}$ MRC-PHE Centre for Environment and Health, Department of Epidemiology and Biostatics, School of Public Health, Imperial College, London, United Kingdom

${ }^{28}$ Department of Community Medicine, Faculty of Health Sciences, UiT The Arctic University of Norway, Troms $\varnothing$, Norway

${ }^{29}$ Cancer Registry of Norway, NO-0304 Oslo, Norway

${ }^{30}$ Department of Medical Epidemiology and Biostatistics, Karolinska Instituet, SE-171 Stockholm, Sweden

${ }^{31}$ Department of Genetic Epidemiology, Folkhälsan Research Center, 00250 Helsinki, Finland

${ }^{32}$ Public Health Directorate, Asturias, Spain

${ }^{33}$ Unit of Nutrition, Environment and Cancer, Cancer Epidemiology Research Program, Catalan Institute of Oncology, Barcelona, Spain

${ }^{34}$ CIBER Epidemiology and Public Health (CIBERESP), Spain

${ }^{35}$ Escuela Andaluza de Salud Pública, Instituto de Investigación Biosanitaria ibs. GRANADA, Hospitales Universitarios de Granada/Universidad de Granada, Granada, Spain

${ }^{36}$ Public Health Direction and CIBERESP-Biodonostia Research Institute, Basque Regional Health Department, San Sebastian, Spain

${ }^{37}$ Department of Epidemiology, Murcia Regional Health Council, IMIB-Arrixaca, Murcia, Spain

${ }^{38}$ Navarre Public Health Institute, Pamplona, Spain

${ }^{39}$ Department of Surgery, Department of Surgical and Perioperative Sciences, Umeå University, Sweden

${ }^{40}$ MRC Epidemiology Unit, Institute of Metabolic Science, Addenbrooke's Hospital, Cambridge, United Kingdom

${ }^{41}$ Clinical Gerontology Unit, Department of Public Health and Primary Care, Institute of Public Health, University of Cambridge, United Kingdom

${ }^{42}$ Cancer Epidemiology Unit, Nuffield Department of Population Health, University of Oxford, Oxford, UK.

${ }^{43}$ Department of Epidemiology and Biostatistics, School of Public Health, Imperial College London, London, United Kingdom

${ }^{44}$ Department of Nutritional Sciences, University of Toronto, Toronto, Canada

* Present address: Section of Medical Statistics, Medical University of Vienna, Spitalgasse 23, 1090 Wien, Austria.

** These authors contributed equally to this work.

\section{Running Title: Advanced Glycation End-Products and Colorectal Cancer Risk}

Keywords: Advanced Glycation End-Products, Colorectal Cancer, European Prospective Investigation into Cancer and Nutrition (EPIC) Cohort

\section{Word Count: 3,201}

Total Number of Tables and Figures: 3 Tables \& 0 Figures 


\section{Financial Support:}

This work was funded by the World Cancer Research Fund (WCRF) International Regular Grant Programme (grant number 2010-251; PI: M. Jenab). This work was also supported in part by grants from the Japan Society for the Promotion of Science (Grant-in-Aid for Scientific Research (B), \#22300264; M. Takeuchi]. The coordination of EPIC is financially supported by the European Commission and the International Agency for Research on Cancer. The national cohorts are supported by Danish Cancer Society (Denmark); Ligue Contre le Cancer; Institut Gustave Roussy; Mutuelle Générale de l'Education Nationale; and Institut National de la Santé et de la Recherche Médicale (INSERM) (France); Deutsche Krebshilfe, Deutsches Krebsforschungszentrum (DKFZ); and Federal Ministry of Education and Research (Germany); Stavros Niarchos Foundation; Hellenic Health Foundation (Greece); Italian Association for Research on Cancer (AIRC); National Research Council; and AIRE-ONLUS Ragusa, AVIS Ragusa, Sicilian Government (Italy); Dutch Ministry of Public Health, Welfare and Sports (VWS); Netherlands Cancer Registry (NKR); LK Research Funds; Dutch Prevention Funds; Dutch ZON (Zorg Onderzoek Nederland); World Cancer Research Fund (WCRF); and Statistics Netherlands (the Netherlands); European Research Council (ERC) and Nordforsk; and Nordic Center of Excellence Programme on Food, Nutrition and Health (Norway); Health Research Fund (FIS); Regional Governments of Andalucía, Asturias, Basque Country, Murcia (No. 6236) and Navarra; and ISCIII RETIC (RD06/0020) and the Catalan Institute of Oncology. (Spain); Swedish Cancer Society; Swedish Scientific Council; and Regional Government of Skåne and Västerbotten (Sweden); Medical Research Council; Stroke Association; British Heart Foundation; Department of Health; Food Standards Agency; and Cancer Research UK/Welcome Trust (UK; K.E. Bradbury and K.T. Khaw).

The funders had no role in study design, data collection and analysis, decision to publish, or preparation of the manuscript.

\section{Joint Correspondence to:}

Mazda Jenab, PhD, Section of Nutrition and Metabolism, International Agency for Research on Cancer

Mailing Address: 150 cours Albert Thomas, 69372 Lyon cedex 08, France

Phone Number: (+33) 4.72.73.80.82

Fax Number: (+33) 4.72.73.83.61

E-mail: jenabm@iarc.fr

W. Robert Bruce, MD, PhD, Department of Nutritional Sciences, University of Toronto

Mailing Address: 150 College Street, Toronto, Ontario, Canada, M5S 3E2

Phone Number: (+1) 416.978.5425

Fax Number: (+1) 416.978 .5882

E-mail: wrbruce@utoronto.ca

\section{Conflict of interest:}

The authors declare that they have no competing or conflict of interests. 


\section{ABSTRACT}

BACKGROUND: A large proportion of colorectal cancers (CRC) are thought to be associated with unhealthy dietary and lifestyle exposures, particularly energy excess, obesity, hyperinsulinemia and hyperglycemia. It has been suggested that these processes stimulate the production of toxic reactive carbonyls from sugars such as glyceraldehyde. Glyceraldehyde contributes to the production of a group of compounds known as glyceraldehyde-derived advanced glycation end-products (glycerAGEs) which may promote CRC through their pro-inflammatory and pro-oxidative properties. The objective of this study nested within a prospective cohort was to explore the association of circulating glycer-AGEs with risk of CRC.

METHODS: 1,055 CRC cases (colon $n=659$; rectal $n=396$ ) were matchced $(1: 1)$ to control subjects. Circulating glycer-AGEs were measured by a competitive enzyme-linked immunosorbent assay. Multivariable conditional logistic regression models were used to calculate odds ratios (OR) and $95 \%$ confidence intervals $(95 \% \mathrm{Cl})$, adjusting for potential confounding factors including smoking, alcohol, physical activity, body mass index, and diabetes status.

RESULTS: Elevated glycer-AGEs levels were not associated with CRC risk (highest vs. lowest quartile, 1.10; $95 \% \mathrm{Cl}, 0.82-1.49)$. Sub-group analyses showed possible divergence by anatomical sub-sites (OR for colon cancer $=0.83 ; 95 \% \mathrm{Cl}, 0.57-1.22 ;$ OR for rectal cancer $=1.90 ; 95 \% \mathrm{Cl}, 1.14-3.19 ; p_{\text {heterogeneity }}=$ $0.14)$.

CONCLUSIONS: In this prospective study, circulating glycer-AGEs were not associated with risk of colon cancer, but showed a positive association with the risk of rectal cancer.

IMPACT: Further research is needed to clarify the role of toxic products of carbohydrate metabolism and energy excess in CRC development.

\section{Word Count: 250}




\section{INTRODUCTION}

Colorectal cancer $(\mathrm{CRC})$ is the third most common cancer in men and the second in women worldwide (1). The incidence of CRC varies approximately 25-fold in different world regions with high risk in developed countries (2). Moreover, it has been observed that the number of new CRC cases is increasing in countries adopting Western dietary and lifestyle patterns, observations which strongly suggest a role for environmental factors in its development (3). Many environmental factors, such as Western-type diet, physical inactivity, and abdominal obesity have been implicated in CRC aetiology (4). Some of the key metabolic consequences of these exposures are hyperinsulinemia, hyperglycemia, inflammation, and oxidative stress, all of which have been proposed as major underlying mechanisms for CRC development $(5,6)$. Interestingly, it has been proposed that interaction between processes of metabolic over-nutrition with inflammation and oxidative stress can lead to the production of reactive carbonyls, a group of highly toxic and possibly carcinogenic compounds, which in turn also have pro-inflammatory and pro-oxidative properties of their own (7). One example of this reaction, which has been well-observed in vitro, is the conversion of glyceraldehyde, an early product of glycolysis, to reactive carbonyls by reactive oxygen species (ROS) (8). Glyceraldehyde contributes to the production of a group of compounds known as

glyceraldehyde-derived advanced glycation end-products (glycer-AGEs) (9). They belong to the larger family of advanced glycation end-products (AGEs), which are stable end-products of the nonenzymatic glycation reaction between reactive carbonyls and free amino groups of proteins, lipids, or nucleic acids $(10,11)$. They can be formed either exogenously in cooking and cigarette smoking processes or endogenously in tissues in the presence of ROS resulting from inflammation or other processes $(12,13)$. Glycer-AGEs are the main component of what is considered the most toxic subgroup of AGEs, also referred to as toxic-AGEs (14). It has been observed that glycer-AGEs can form in vivo from both reactive carbonyls and as a direct consequence of sugar metabolism $(15,16)$. Thus, it is plausible that the circulating concentration of glycer-AGEs, aside from having direct inflammatory 
and oxidative properties, may also be an indicator of the extent of direct exposures to reactive carbonyl species.

AGEs in general have been implicated in the development of diabetes $(17,18)$ and in ocular (19), renal (20), cardiovascular (21), and some neurodegenerative disorders (22), as well as in several cancers $(23,24)$. For their part, glycer-AGEs have been shown to be cytotoxic in vitro $(25,26)$ and findings from animal studies suggest involvement in the pathogenesis of insulin resistance and diabetes (27) as well as its complications (28). Human studies suggest the involvement of glycer-AGEs in the development of Alzheimer's disease (29), non-alcoholic steatohepatitis (30), vascular inflammation (31) and some rare disorders $(32,33)$. In addition to their potential direct effects, some evidence indicates that glycer-AGEs interact strongly with the receptor for AGEs (RAGE) to cause inflammatory and oxidative responses $(28,34)$ - which are suspected in the development of various cancers, including CRC (35-37). In view of their apparent direct cytotoxicity, adverse effects on cell function and involvement in inflammatory and oxidative processes, a role for glycer-AGEs in CRC development is plausible, but has yet to be examined in studies on humans. In this study, nested within the large multi-national European Prospective Investigation into Cancer and Nutrition (EPIC) cohort, we investigated the association of circulating concentrations of glycer-AGEs with risk of CRC. We hypothesize that higher concentrations of glycer-AGEs would be positively associated with risk of CRC development.

\section{MATERIALS AND METHODS}

\section{Study population and data collection}

We used a case-control design nested within the EPIC cohort, a large prospective cohort study with over 520,000 subjects enrolled from 23 centres in 10 Western European countries (Denmark, France, Greece, Germany, Italy, Netherlands, Norway, Spain, Sweden, and United Kingdom). The rationale and methods of EPIC, including information on dietary assessment methods, blood collection protocols, and follow-up procedures, have been previously reviewed (38). Briefly, 
individuals who were eligible for the study were selected from the general population of a specific geographical area, town, or province. Exceptions included the French sub-cohort, which is based on members of the health insurance system or state-school employees, the Utrecht (Netherlands) subcohort, which is based on women who underwent screening for breast cancer, and the Oxford (UK) sub-cohort, which targeted recruitment towards health-conscious people, including vegetarians. Between 1992 and 1998, standardized lifestyle and personal history questionnaires, anthropometric data, and blood samples were collected from most participants at recruitment. Diet over the previous 12 months was assessed at recruitment by validated country-specific questionnaires designed to ensure high compliance and improved measures of local dietary habits (39). In each of the study centres, either fasting or non-fasting blood samples of at least $30 \mathrm{~mL}$ were drawn from those participants who provided a blood sample and stored at $5^{\circ} \mathrm{C}$ to $10^{\circ} \mathrm{C}$, protected from light, and transported to local laboratories for processing and aliquoting as previously described $(38,39)$. In all countries, except Denmark and Sweden, blood was separated in the local EPIC centres and stored at the International Agency for Research on Cancer (Lyon, France; $-196^{\circ} \mathrm{C}$, nitrogen vapour). In Denmark, blood samples were stored locally at $-150^{\circ} \mathrm{C}$ under nitrogen vapour. In Sweden, samples were stored in $-80^{\circ} \mathrm{C}$ freezers.

\section{Follow-up for cancer incidence and vital status}

Vital status follow-up ( $98.4 \%$ complete) is collected by record linkage with regional and/or national mortality registries in all countries except Germany and Greece, and the Italian centre of Naples, where data are collected actively. Cancer incidence is determined through record linkage with regional cancer registries (Denmark, other Italian centres, the Netherlands, Norway, Spain, Sweden, and United Kingdom; for this analysis complete up to June 2003) or via a combination of methods, including linkage with health insurance records, contacts with cancer and pathology registries, and active follow-up through study subjects or their next-of-kin (France, Germany, and Greece; for this analysis complete up to June 2002). 


\section{Nested case-control study design and selection of study subjects}

\section{Case ascertainment and selection}

Colon cancers were defined as incident tumours in the cecum, appendix, ascending colon, hepatic flexure, transverse colon, splenic flexure, and descending and sigmoid (C18.0 - C18.7, according to the $10^{\text {th }}$ Revision of the International Statistical Classification of Diseases, Injury, and Cause of Death), as well as tumours that were overlapping or unspecified (C18.8 and C18.9). Rectal cancers were defined as incident tumours occurring at the recto-sigmoid junction (C19) or rectum (C20). Subjects with anal canal tumours were excluded from the study. CRC is defined as a combination of the colon and rectal cancer cases.

After exclusions (225 cases for insufficient remaining bio-sample, 26 cases for missing laboratory values of glycer-AGEs and 29 cases with incomplete matching), a total of 1,055 first incident $C R C$ cases (colon $n=659$; rectal $n=396$ ) were identified.

Control selection

For selection of control subjects, an incidence density sampling protocol was applied. For each case one control subject was chosen at random among appropriate risk sets consisting of all cohort members alive and free of cancer (except nonmelanoma skin cancer) at the time of diagnosis of the index case. Matching characteristics were study center (to account for centre-specific differences such as questionnaire design and blood collection procedures), sex, age, time of blood collection and fasting status at the time of blood collection (less than 3 hours, 3-6 hours, and more than 6 hours). Women were also matched on menopausal status (premenopausal, peri-menopausal, postmenopausal, or surgically menopausal). Premenopausal women were matched on phase of the menstrual cycle at blood collection, and postmenopausal women were matched on current use of hormone replacement therapy.

\section{Laboratory analyses}

Serum levels of glycer-AGEs were measured with a competitive enzyme-linked immunosorbent assay (ELISA) at Kanazawa Medical University, Japan by using immunopurified 
glycer-AGEs antibody as described previously (15). Briefly, 96-well microtiter plates were coated with $1 \mu \mathrm{g} / \mathrm{mL}$ glycer-AGEs to each well and incubated overnight in a cold room. Wells were washed three times with $0.3 \mathrm{~mL}$ of phosphate-buffered saline (PBS)-Tween-20 (PBS-Tween-20). Wells were then blocked by incubation for 1 hour with $0.2 \mathrm{~mL}$ of a solution of PBS containing $1 \%$ bovine serum albumin (BSA). After washing with PBS-Tween-20, test samples (50 $\mu \mathrm{L})$ were added to each well as a competitor for $50 \mu \mathrm{L}$ of glycer-AGEs antibody (1:1,000), followed by incubation for 2 hours at $30^{\circ} \mathrm{C}$ with gentle shaking on a horizontal rotary shaker. Wells then were washed with PBS-Tween-20 and developed with an alkaline phosphatase-linked anti-rabbit lgG utilizing p-nitrophenyl phosphate as the colorimetric substrate. Results are expressed as glycer-AGEs units (U) per millilitre $(\mathrm{mL})$ of serum, with $1 \mathrm{U}$ corresponding to $1 \mu \mathrm{g}$ of glycer-AGEs standard. Sensitivity and intra- and inter-assay coefficients of variation were $0.01 \mathrm{U} / \mathrm{mL}, 6.2$ and $8.8 \%$, respectively $(31,40)$. For all analyses, laboratory technicians were blinded to the case-control status of the samples and cases and matched controls were run on the same plate.

Some existing biomarker measures on the same cases and matched controls were also utilized for this study. Measurements of glycated haemoglobin (HbA1c) were previously run on erythrocyte hemolysate using high performance liquid chromatography method (Bio-Rad Variant II instrument, Bio Lad Laboratories, Hercules, California) with intra-batch coefficient of variations of $2.5 \%$ (41). High-sensitivity C-reactive protein (hs-CRP) and high-density lipoprotein (HDL) cholesterol concentrations were measured using a high-sensitivity assay (Beckman-Coulter, Woerden, the Netherlands) and a colorimetric method, respectively, on a Synchron LX-20 Pro auto-analyzer (Beckman-Coulter). The inter-assay coefficients of variation were $6.0 \%-6.5 \%$ and $3.4 \%-4.1 \%$ at various concentrations of hs-CRP and HDL cholesterol, respectively (42).

\section{Statistical analysis}

The distributions of selected baseline demographic and dietary characteristics between colon and rectal cancer cases and the matched controls were described. 
Conditional logistic regression was used to estimate the odds ratios (OR) and $95 \%$ confidence intervals $(\mathrm{Cl})$ of $\mathrm{CRC}$, and by anatomical sub-site of cancers of the colon and rectum in relation to levels of circulating glycer-AGEs. Glycer-AGEs levels were ranked into quartiles whose cut-points were determined based on the distribution among the controls with the lowest quartile as the reference category.

Risk estimates were computed as both univariate analyses based on the matching factors, and multivariable analyses, with additional adjustments for potential confounders including smoking status (status/duration/intensity of smoking), body mass index (BMI, $\left.\mathrm{kg} / \mathrm{m}^{2}\right)$, education level (as an indicator variable for socioeconomic status), total alcohol consumption (g/day), physical activity (combined recreational and household activity; expressed as sex-specific categories of metabolic equivalents), total energy intake ( $\mathrm{kcal} /$ day), total daily intakes of fibre (g/day), fruits and vegetable (g/day), and red/processed meats (g/day) (43-48), and diabetes status $(49,50)$. Subjects were classified as diabetic if they had baseline $\mathrm{HbA} 1 \mathrm{c}$ levels $\geq 6.5 \%$ and/or self-reported diabetes at recruitment $(n=174)$. For all models, collinearity was assessed and tests for linear trend were performed using a score variable with values from 1 to 4 , consistent with the quartile grouping. Statistical tests for heterogeneity to test whether the associations differ by anatomical sub-sites of colon and rectal cancer were based on $\chi^{2}$ statistics.

We also assessed effect modification by several factors; sex and tumour location were hypothesised as effect modifiers at the time of study design because of their modifying effect of some $\mathrm{CRC}$ risk factors in previous studies (4), while smoking, alcohol, and BMI were examined for hypothesis generation. The product term of glycer-AGEs (in quartiles) and each potential effect modifier was included in the model, and evaluated by a likelihood ratio test. In the sensitivity analysis, we repeated the main multivariable-adjusted models after excluding cases that occurred in the first 2 years of the follow-up ( $n=142$ for colon and $n=82$ for rectum) and their matched controls to avoid possible reverse causality, and subjects with diabetes and their matched cases and controls $(n=180$ for colon and $n=128$ for rectum). Heterogeneity tests were based on $\chi^{2}$ statistics. 
Logistic restricted cubic spline models were used to explore possible deviation from nonlinear relationships between glycer-AGEs and CRC, with 4 knots specific at the median of each quartile of glycer-AGEs levels.

A two-tailed $p$-value $<0.05$ was considered to be statistically significant. All statistical analyses were performed with SAS version 9.3 (SAS Institute, Cary, NC) statistical software package.

\section{RESULTS}

\section{Baseline characteristics of cases and controls}

Selected baseline characteristics of the colon and rectal cancer cases and their matched controls are compared in Table 1. Colon and rectal cancer cases were on average 59 years and 58 years old and had mean follow-up times of 3.7 and 3.9 years, respectively. Colon cancer cases included a higher proportion of individuals who reported being physically inactive ( $15.8 \%$ vs. $11.5 \%)$, had higher average hs-CRP levels ( $3.1 \mathrm{vs.} 2.2 \mathrm{mg} / \mathrm{l})$, and consumed less fruits and vegetables ( $369 \mathrm{~g} / \mathrm{d}$ vs. $418 \mathrm{~g} / \mathrm{d}$ ) than their matched controls. Both colon and rectal cancer cases were more likely to be diabetic ( $10.2 \%$ vs. $6.9 \%$ for colon and $11.7 \%$ vs. $8.0 \%$ for rectal cancer) than their counterparts. No significant case-control differences were observed in other baseline characteristics.

\section{Associations of glycer-AGEs with CRC}

Table 2 presents ORs and 95\% Cls for the association between glycer-AGEs levels, in quartiles using the lowest category as a referent, and CRC. Overall, models with adjustments for matching factors showed very similar results with the multivariable models with adjustments for established confounding factors in addition to matching factors. Elevated glycer-AGEs levels were not associated with $\mathrm{CRC}$ risk (multivariable adjusted $\mathrm{OR}=1.10 ; 95 \% \mathrm{Cl}, 0.82-1.49$, comparing highest vs. lowest quartiles; $\left.p_{\text {trend }}=0.87\right)$. When analyses were run separately for CRC sub-sites, no association was observed between glycer-AGEs and risk of colon cancer (multivariable adjusted $\mathrm{OR}=0.83 ; 95 \% \mathrm{Cl}$, 0.57-1.22; $\mathrm{p}_{\text {trend }}=0.25$ ), while a statistically significant positive association was observed with rectal 
cancer (multivariable adjusted $\mathrm{OR}=1.90 ; 95 \% \mathrm{Cl}, 1.14-3.19 ; \mathrm{p}_{\text {trend }}=0.04$ ); although the test for heterogeneity was not statistically significant $\left(P_{\text {heterogeneity }}=0.14\right)$.

After testing potential effect modification by various factors relevant for CRC risk and glycerAGEs concentrations, alcohol consumption showed a statistically significantly modifying effect on the association between glycer-AGEs level and rectal cancer $\left(p_{\text {interaction }}=0.03\right)$. Further stratification by level of alcohol consumption (dichotomized based on the sex-specific median values of total alcohol consumption among controls; men, $18.1 \mathrm{~g} / \mathrm{d}$; women, $5.7 \mathrm{~g} / \mathrm{d}$ ) showed a significant, positive association for rectal cancer among high alcohol consumers (> median) group (multivariate adjusted $\left.\mathrm{OR}=2.70 ; 95 \% \mathrm{Cl}, 1.29-5.62 ; p_{\text {trend }}=0.01\right)$ (Table 3). No significant effect modifications, including alcohol consumption, were observed for colon cancer.

\section{Sensitivity analysis}

After exclusion of cases which occurred during the first two years of follow-up and their matched controls, the overall findings did not change substantially for either of the CRC, colon or rectum anatomical sites. The associations between glycer-AGEs and CRC, colon, and rectal cancer risks were also similar after excluding subjects with diabetes. Spline models confirmed that associations between glycer-AGEs and risk of colon or rectal cancers were linear.

\section{DISCUSSION}

In this nested case-control study within the large prospective EPIC cohort, we did not observe any overall association between increasing circulating levels of glycer-AGEs and CRC. However, sub-group analyses by anatomical sub-site showed a statistically significant positive association with risk of rectal cancer, particularly among those with higher alcohol consumption. We found no association between glycer-AGEs and colon cancer risk.

A role for AGEs in CRC development has been hypothesized (37) but not yet fully explored. This may be due to the complexity of this family of compounds, whose heterogeneous structures are far from being fully understood (51). Of the handful of different AGEs species identified, $N$ - 
(carboxymethyl)-lysine (CML) is probably among the most studied and is inferred as an indicator of overall AGEs exposure. The only evidence to date from prospective studies on blood CML measures shows no association with risk of CRC (36) or pancreatic cancer (52), results which are in line with our present findings for CRC, although we studied a different and possibly more toxic species of AGEs (14).

Our sub-group observations of a risk for rectal but not colon cancer are interesting. One explanation is chance, given the limited sample size of the rectal cancer sub-group and the nonstatistically significant test for heterogeneity of effect between the anatomical sub-sites. However, there are some possible biologic explanations for this observation: (a) Colon and rectal tissues may differ in expression level of the receptors that either bind AGEs (i.e. RAGE) and then elicit various pro-tumorigenic effects (53), or those that act as competitive inhibitors of RAGE-mediated signalling pathways, i.e. soluble form of RAGE (sRAGE) (54). Glycer-AGEs have been shown to have high binding affinity for RAGE (34), and decreased circulating sRAGE levels have been observed in CRC (36). If RAGE and SRAGE expression and activity levels differ between colon and rectal tissues, then a difference of effect associated with AGEs exposure may be plausible. In the current literature, there is some evidence indicating an increase in RAGE expression in human colon tissues in Crohn's disease (55), and in the colon tissue of diabetic rats (56), but direct comparisons of human colon versus rectal normal and tumour tissues have not been reported and would warrant further study. (b) AGEsinduced effects and AGEs accumulation may vary in tumors from different anatomical sites (24), resulting in tissue-specific effects of glycer-AGEs or AGEs in general. And (c) colon and rectal tumours differ by gene mutations (e.g. $K$-ras and $A P C$ gene) and biological behaviour (57-59), indicating that they may arise from different mechanisms of carcinogenesis and hence be differentially affected by various endogenous and exogenous factors, such as AGEs. Thus, although they are plausible, our findings of a differential association between the colon and rectal anatomical sub-sites require both replication and further study. 
A related incidental observation in our sub-group analyses was a statistically significant effect modification of alcohol on the association between glycer-AGEs and rectal cancer risk. Although alcoholic beverages may be important exogenous sources of AGEs (60) and several studies, including our own (48), have observed a stronger association between higher alcohol consumption and development of rectal than colon cancer $(61,62)$, these observations do not explain the modifying effect of alcohol on the association of AGEs with rectal cancer that we observed. The reasons for this modification are therefore unclear and, if the modification were replicated, would warrant further study.

The present study has several strengths. The foremost is the prospective design and the prediagnostic collection of dietary/lifestyle information and blood samples from the cohort participants. Our study was also large and well powered to explore associations with CRC, but size was a limiting factor in our sub-group analyses. A key limitation of our study is that we only had a single measure of glycer-AGEs, taken at time of recruitment into the cohort (baseline). While there is no information as to what extent a single measure of AGEs reflects long term exposure, random errors in measuring long term exposure would be expected to reduce any observed disease risk associations towards the null. The finding of a significant association between glycer-AGEs and the risk of rectal cancer suggests that any measurement error was not sufficient to obscure the association, though the relative risks we observe may underestimate the strength of the true association.

In summary, in this prospective study in European populations, circulating glycer-AGEs were not associated with overall risk of CRC. Further research is needed to investigate the role of glyerAGEs and AGEs in general in CRC development. 
Author Manuscript Published OnlineFirst on September 24, 2015; DOI: 10.1158/1055-9965.EPI-15-0422

Author manuscripts have been peer reviewed and accepted for publication but have not yet been edited.

\section{REFERENCES}

1. Jemal A, Bray F, Center MM, Ferlay J, Ward E, Forman D. Global cancer statistics. CA: a cancer journal for clinicians. 2011;61:69-90.

2. Ferlay J, Shin HR, Bray F, Forman D, Mathers C, Parkin DM. Estimates of worldwide burden of cancer in 2008: GLOBOCAN 2008. International journal of cancer Journal international du cancer. 2010;127:2893-917.

3. Durko L, Malecka-Panas E. Lifestyle Modifications and Colorectal Cancer. Current colorectal cancer reports. 2014;10:45-54.

4. Colorectal Cancer 2011 Report: Food, Nutrition, Physical Activity, and the Prevention of Colorectal Cancer: World Cancer Research Fund / American Institute for Cancer Research; 2011.

5. Giovannucci E. Metabolic syndrome, hyperinsulinemia, and colon cancer: a review. The American journal of clinical nutrition. 2007;86:s836-42.

6. Siddiqui AA. Metabolic syndrome and its association with colorectal cancer: a review. The American journal of the medical sciences. 2011;341:227-31.

7. Cai W, Gao QD, Zhu L, Peppa M, He C, Vlassara H. Oxidative stress-inducing carbonyl compounds from common foods: novel mediators of cellular dysfunction. Molecular medicine. 2002;8:337-46.

8. Benov L. Short chain sugars as endogenous toxins. Chapter 6 In Endogenous Toxins. In: O'Brien PJaB, W. Robert editor. Weinheim: Wiley-VCH; 2010. p. 153-71.

9. Vlassara H, Bucala R, Striker L. Pathogenic effects of advanced glycosylation: biochemical, biologic, and clinical implications for diabetes and aging. Laboratory investigation; a journal of technical methods and pathology. 1994;70:138-51.

10. Goldin A, Beckman JA, Schmidt AM, Creager MA. Advanced glycation end products: sparking the development of diabetic vascular injury. Circulation. 2006;114:597-605.

11. Singh R, Barden A, Mori T, Beilin L. Advanced glycation end-products: a review. Diabetologia. 2001;44:129-46.

12. Goldberg T, Cai W, Peppa M, Dardaine V, Baliga BS, Uribarri J, et al. Advanced glycoxidation end products in commonly consumed foods. Journal of the American Dietetic Association.

2004;104:1287-91.

13. Nicholl ID, Bucala R. Advanced glycation endproducts and cigarette smoking. Cellular and molecular biology. 1998;44:1025-33.

14. Takeuchi M, Yamagishi S. TAGE (toxic AGEs) hypothesis in various chronic diseases. Medical hypotheses. 2004;63:449-52.

15. Takeuchi M, Makita Z, Bucala R, Suzuki T, Koike T, Kameda Y. Immunological evidence that non-carboxymethyllysine advanced glycation end-products are produced from short chain sugars and dicarbonyl compounds in vivo. Molecular medicine. 2000;6:114-25.

16. Usui T, Shimohira K, Watanabe H, Hayase F. Detection and determination of glyceraldehydederived pyridinium-type advanced glycation end product in streptozotocin-induced diabetic rats. Bioscience, biotechnology, and biochemistry. 2007;71:442-8.

17. Yamagishi S, Takeuchi M, Inagaki Y, Nakamura K, Imaizumi T. Role of advanced glycation end products (AGEs) and their receptor (RAGE) in the pathogenesis of diabetic microangiopathy. International journal of clinical pharmacology research. 2003;23:129-34.

18. Goh SY, Cooper ME. Clinical review: The role of advanced glycation end products in progression and complications of diabetes. The Journal of clinical endocrinology and metabolism. 2008;93:1143-52.

19. Kandarakis SA, Piperi C, Topouzis F, Papavassiliou AG. Emerging role of advanced glycationend products (AGEs) in the pathobiology of eye diseases. Progress in retinal and eye research. 2014;42:85-102.

20. lacobini C, Menini S, Ricci C, Scipioni A, Sansoni V, Mazzitelli G, et al. Advanced lipoxidation end-products mediate lipid-induced glomerular injury: role of receptor-mediated mechanisms. The Journal of pathology. 2009;218:360-9. 
Author Manuscript Published OnlineFirst on September 24, 2015; DOI: 10.1158/1055-9965.EPI-15-0422

Author manuscripts have been peer reviewed and accepted for publication but have not yet been edited.

21. Del Turco S, Basta G. An update on advanced glycation endproducts and atherosclerosis. BioFactors. 2012;38:266-74.

22. Li J, Liu D, Sun L, Lu Y, Zhang Z. Advanced glycation end products and neurodegenerative diseases: mechanisms and perspective. Journal of the neurological sciences. 2012;317:1-5.

23. Chen H, Wu L, Li Y, Meng J, Lin N, Yang D, et al. Advanced glycation end products increase carbohydrate responsive element binding protein expression and promote cancer cell proliferation. Molecular and cellular endocrinology. 2014;395:69-78.

24. van Heijst JW, Niessen HW, Hoekman K, Schalkwijk CG. Advanced glycation end products in human cancer tissues: detection of Nepsilon-(carboxymethyl)lysine and argpyrimidine. Annals of the New York Academy of Sciences. 2005;1043:725-33.

25. Takino J, Kobayashi Y, Takeuchi M. The formation of intracellular glyceraldehyde-derived advanced glycation end-products and cytotoxicity. Journal of gastroenterology. 2010;45:646-55.

26. Usui T, Shizuuchi S, Watanabe H, Hayase F. Cytotoxicity and oxidative stress induced by the glyceraldehyde-related Maillard reaction products for HL-60 cells. Bioscience, biotechnology, and biochemistry. 2004;68:333-40.

27. Ebata Y, Takino J, Tsuchiya H, Sakabe T, Ikeda Y, Hama S, et al. Presence of glyceraldehydederived advanced glycation end-products in the liver of insulin-resistant mice. International journal for vitamin and nutrition research Internationale Zeitschrift fur Vitamin- und Ernahrungsforschung Journal international de vitaminologie et de nutrition. 2013;83:137-41.

28. Takeuchi M, Takino J, Yamagishi S. Involvement of TAGE-RAGE System in the Pathogenesis of Diabetic Retinopathy. Journal of ophthalmology. 2010;2010:170393.

29. Choei H, Sasaki N, Takeuchi M, Yoshida T, Ukai W, Yamagishi S, et al. Glyceraldehyde-derived advanced glycation end products in Alzheimer's disease. Acta neuropathologica. 2004;108:189-93.

30. Hyogo H, Yamagishi S, Iwamoto K, Arihiro K, Takeuchi M, Sato T, et al. Elevated levels of serum advanced glycation end products in patients with non-alcoholic steatohepatitis. Journal of gastroenterology and hepatology. 2007;22:1112-9.

31. Tahara N, Yamagishi S, Takeuchi M, Honda A, Tahara A, Nitta Y, et al. Positive association between serum level of glyceraldehyde-derived advanced glycation end products and vascular inflammation evaluated by [(18)F]fluorodeoxyglucose positron emission tomography. Diabetes care. 2012;35:2618-25.

32. Kitamura M, Kitaichi N, Takeuchi M, Kitamei H, Namba K, Yamagishi SI, et al. Decrease in the glyceraldehyde derived advanced glycation end products in the sera of patients with Vogt-KoyanagiHarada disease. The British journal of ophthalmology. 2005;89:1407-9.

33. Dong Z, Iwata D, Kitaichi N, Takeuchi M, Sato M, Endo N, et al. Amelioration of experimental autoimmune uveoretinitis by inhibition of glyceraldehyde-derived advanced glycation end-product formation. Journal of leukocyte biology. 2014;96:1077-85.

34. Yamamoto Y, Yonekura H, Watanabe T, Sakurai S, Li H, Harashima A, et al. Short-chain aldehyde-derived ligands for RAGE and their actions on endothelial cells. Diabetes research and clinical practice. 2007;77 Suppl 1:S30-40.

35. Grote VA, Nieters A, Kaaks R, Tjonneland A, Roswall N, Overvad K, et al. The associations of advanced glycation end products and its soluble receptor with pancreatic cancer risk: a case-control study within the prospective EPIC Cohort. Cancer epidemiology, biomarkers \& prevention : a publication of the American Association for Cancer Research, cosponsored by the American Society of Preventive Oncology. 2012;21:619-28.

36. Jiao L, Taylor PR, Weinstein SJ, Graubard BI, Virtamo J, Albanes D, et al. Advanced glycation end products, soluble receptor for advanced glycation end products, and risk of colorectal cancer.

Cancer epidemiology, biomarkers \& prevention : a publication of the American Association for Cancer Research, cosponsored by the American Society of Preventive Oncology. 2011;20:1430-8.

37. Yamagishi S, Nakamura K, Inoue H, Kikuchi S, Takeuchi M. Possible participation of advanced glycation end products in the pathogenesis of colorectal cancer in diabetic patients. Medical hypotheses. 2005;64:1208-10. 
Author Manuscript Published OnlineFirst on September 24, 2015; DOI: 10.1158/1055-9965.EPI-15-0422

Author manuscripts have been peer reviewed and accepted for publication but have not yet been edited.

38. Riboli E, Kaaks R. The EPIC Project: rationale and study design. European Prospective Investigation into Cancer and Nutrition. Int J Epidemiol. 1997;26 Suppl 1:S6-14.

39. Riboli E, Hunt KJ, Slimani N, Ferrari P, Norat T, Fahey M, et al. European Prospective Investigation into Cancer and Nutrition (EPIC): study populations and data collection. Public health nutrition. 2002;5:1113-24.

40. Jinno M, Takeuchi M, Watanabe A, Teruya K, Hirohama J, Eguchi N, et al. Advanced glycation end-products accumulation compromises embryonic development and achievement of pregnancy by assisted reproductive technology. Hum Reprod. 2011;26:604-10.

41. Rinaldi S, Rohrmann S, Jenab M, Biessy C, Sieri S, Palli D, et al. Glycosylated hemoglobin and risk of colorectal cancer in men and women, the European prospective investigation into cancer and nutrition. Cancer Epidemiol Biomarkers Prev. 2008;17:3108-15.

42. Aleksandrova K, Jenab M, Boeing H, Jansen E, Bueno-de-Mesquita HB, Rinaldi S, et al. Circulating C-reactive protein concentrations and risks of colon and rectal cancer: a nested casecontrol study within the European Prospective Investigation into Cancer and Nutrition. American journal of epidemiology. 2010;172:407-18.

43. Murphy N, Norat T, Ferrari P, Jenab M, Bueno-de-Mesquita B, Skeie G, et al. Dietary fibre intake and risks of cancers of the colon and rectum in the European prospective investigation into cancer and nutrition (EPIC). PLoS One. 2012;7:e39361.

44. van Duijnhoven FJ, Bueno-De-Mesquita HB, Ferrari P, Jenab M, Boshuizen HC, Ros MM, et al. Fruit, vegetables, and colorectal cancer risk: the European Prospective Investigation into Cancer and Nutrition. The American journal of clinical nutrition. 2009;89:1441-52.

45. Norat T, Bingham S, Ferrari P, Slimani N, Jenab M, Mazuir M, et al. Meat, fish, and colorectal cancer risk: the European Prospective Investigation into cancer and nutrition. J Natl Cancer Inst. 2005;97:906-16.

46. Leufkens AM, Van Duijnhoven FJ, Siersema PD, Boshuizen HC, Vrieling A, Agudo A, et al. Cigarette smoking and colorectal cancer risk in the European Prospective Investigation into Cancer and Nutrition study. Clin Gastroenterol Hepatol. 2011;9:137-44.

47. Steins Bisschop CN, van Gils CH, Emaus MJ, Bueno-de-Mesquita HB, Monninkhof EM, Boeing $\mathrm{H}$, et al. Weight change later in life and colon and rectal cancer risk in participants in the EPICPANACEA study1,3. The American journal of clinical nutrition. 2014;99:139-47.

48. Ferrari P, Jenab M, Norat T, Moskal A, Slimani N, Olsen A, et al. Lifetime and baseline alcohol intake and risk of colon and rectal cancers in the European prospective investigation into cancer and nutrition (EPIC). International journal of cancer Journal international du cancer. 2007;121:2065-72.

49. Berster JM, Goke B. Type 2 diabetes mellitus as risk factor for colorectal cancer. Archives of physiology and biochemistry. 2008;114:84-98.

50. Ahmed N. Advanced glycation endproducts--role in pathology of diabetic complications. Diabetes research and clinical practice. 2005;67:3-21.

51. Gkogkolou P, Bohm M. Advanced glycation end products: Key players in skin aging? Dermatoendocrinology. 2012;4:259-70.

52. Jiao L, Weinstein SJ, Albanes D, Taylor PR, Graubard BI, Virtamo J, et al. Evidence that serum levels of the soluble receptor for advanced glycation end products are inversely associated with pancreatic cancer risk: a prospective study. Cancer research. 2011;71:3582-9.

53. Yan SF, Ramasamy R, Naka Y, Schmidt AM. Glycation, inflammation, and RAGE: a scaffold for the macrovascular complications of diabetes and beyond. Circulation research. 2003;93:1159-69.

54. Vazzana N, Santilli F, Cuccurullo C, Davi G. Soluble forms of RAGE in internal medicine. Internal and emergency medicine. 2009;4:389-401.

55. Ciccocioppo R, Vanoli A, Klersy C, Imbesi V, Boccaccio V, Manca R, et al. Role of the advanced glycation end products receptor in Crohn's disease inflammation. World journal of gastroenterology : WJG. 2013;19:8269-81.

56. Chen P, Zhao J, Gregersen H. Up-regulated expression of advanced glycation end-products and their receptor in the small intestine and colon of diabetic rats. Digestive diseases and sciences. 2012;57:48-57. 
57. Frattini M, Balestra D, Suardi S, Oggionni M, Alberici P, Radice P, et al. Different genetic features associated with colon and rectal carcinogenesis. Clinical cancer research : an official journal of the American Association for Cancer Research. 2004;10:4015-21.

58. Li FY, Lai MD. Colorectal cancer, one entity or three. Journal of Zhejiang University Science B. 2009;10:219-29.

59. Kapiteijn E, Liefers GJ, Los LC, Kranenbarg EK, Hermans J, Tollenaar RA, et al. Mechanisms of oncogenesis in colon versus rectal cancer. The Journal of pathology. 2001;195:171-8.

60. Kalousova M, Zima T, Tesar V, Stipek S, Sulkova S. Advanced glycation end products in clinical nephrology. Kidney \& blood pressure research. 2004;27:18-28.

61. Bongaerts BW, van den Brandt PA, Goldbohm RA, de Goeij AF, Weijenberg MP. Alcohol consumption, type of alcoholic beverage and risk of colorectal cancer at specific subsites.

International journal of cancer Journal international du cancer. 2008;123:2411-7.

62. Otani T, Iwasaki M, Yamamoto S, Sobue T, Hanaoka T, Inoue M, et al. Alcohol consumption, smoking, and subsequent risk of colorectal cancer in middle-aged and elderly Japanese men and women: Japan Public Health Center-based prospective study. Cancer epidemiology, biomarkers \& prevention : a publication of the American Association for Cancer Research, cosponsored by the American Society of Preventive Oncology. 2003;12:1492-500. 
Table 1: Description of cases and matched controls, by anatomical site

\begin{tabular}{|c|c|c|c|c|}
\hline \multirow[t]{2}{*}{ Characteristics } & \multicolumn{2}{|c|}{ Colon Cancer } & \multicolumn{2}{|c|}{ Rectal Cancer } \\
\hline & Cases & Matched Controls & Cases & Matched Controls \\
\hline Men, $\mathrm{n}$ & 297 & 297 & 213 & 213 \\
\hline Women, $\mathrm{n}$ & 362 & 362 & 183 & 183 \\
\hline Total & 659 & 659 & 396 & 396 \\
\hline \multicolumn{5}{|l|}{ Age, years, mean (SD) } \\
\hline At recruitment & $58.8(7.2)$ & $58.8(7.2)$ & $58.1(6.8)$ & $57.7(6.6)$ \\
\hline At blood collection & $59.0(7.2)$ & $58.9(7.2)$ & $58.1(6.8)$ & $57.8(6.5)$ \\
\hline Body mass index, $\mathrm{kg} / \mathrm{m}^{2}$, mean (SD) & $26.8(4.5)$ & $26.3(3.9)$ & $26.6(4.0)$ & $26.4(3.8)$ \\
\hline Waist circumference, $\mathrm{cm}$, mean (SD) & $90.4(13.3)$ & $88.0(12.1)$ & $90.4(12.9)$ & $89.8(12.9)$ \\
\hline \multicolumn{5}{|l|}{ Smoking status/duration/intensity, n (\%) } \\
\hline Never-smokers & $276(41.9)$ & $291(44.2)$ & $155(39.1)$ & $156(39.4)$ \\
\hline Ex-smokers, duration of smoking $<10$ years & $40(6.1)$ & $43(6.5)$ & $21(5.3)$ & $29(7.3)$ \\
\hline Ex-smokers, duration of smoking $\geq 10$ years & $160(24.3)$ & $165(25.0)$ & $103(26.0)$ & $93(23.5)$ \\
\hline Ex-smokers, missing duration of smoking & $15(2.3)$ & $12(1.8)$ & $4(1.0)$ & $8(2.0)$ \\
\hline Smokers, $<15$ cigarettes a day & $110(16.7)$ & $96(14.6)$ & 79 (19.9) & $65(16.4)$ \\
\hline Smokers, $\geq 15$ to $<25$ cigarettes a day & $41(6.2)$ & $39(5.9)$ & $24(6.1)$ & $34(8.6)$ \\
\hline Smokers, $\geq 25$ cigarettes a day & $9(1.4)$ & $6(0.9)$ & $7(1.8)$ & $5(1.3)$ \\
\hline Missing smoking status & $8(1.2)$ & $7(1.1)$ & $3(0.8)$ & $6(1.5)$ \\
\hline \multicolumn{5}{|l|}{ Physical activity, n (\%) } \\
\hline Inactive & $104(15.8)$ & $76(11.5)$ & $57(14.4)$ & $58(14.6)$ \\
\hline Moderately inactive & $201(30.5)$ & $206(31.3)$ & $115(29.0)$ & $99(25.0)$ \\
\hline Moderately active & $288(43.7)$ & $293(44.5)$ & $176(44.4)$ & $169(42.7)$ \\
\hline Active & $62(9.4)$ & $78(11.8)$ & $48(12.1)$ & $61(15.4)$ \\
\hline Missing/unspecified & $4(0.6)$ & $6(0.9)$ & 0 & $9(2.3)$ \\
\hline \multicolumn{5}{|l|}{ Education level, n (\%) } \\
\hline None/primary & $256(38.8)$ & $288(43.7)$ & $147(37.1)$ & $163(41.2)$ \\
\hline Technical/professional & $151(22.9)$ & $161(24.4)$ & $108(27.3)$ & $110(27.8)$ \\
\hline Secondary & $113(17.1)$ & $83(12.6)$ & $54(13.6)$ & $41(10.3)$ \\
\hline University or higher & $117(17.7)$ & $109(16.5)$ & 75 (18.9) & 75 (18.9) \\
\hline Missing/unspecified & $22(3.3)$ & $18(2.7)$ & $12(3.0)$ & $7(1.8)$ \\
\hline \multicolumn{5}{|l|}{ Diabetes status, \% } \\
\hline Self-reported diabetes at recruitment, n (\%) & $29(5.3)$ & $29(5.3)$ & $24(6.9)$ & $16(4.6)$ \\
\hline Subjects $\mathrm{HbA} 1 \mathrm{c} \geq 6.5 \%, \mathrm{n}(\%)$ & $54(10.0)$ & $25(6.1)$ & $36(11.1)$ & $22(8.6)$ \\
\hline
\end{tabular}




\begin{tabular}{llll}
\hline $\begin{array}{l}\text { Self-reported diabetes or HbA1c } \geq 6.5 \%, \mathrm{n}(\%) \\
\text { Dietary variables (g/day, median (IQR)) }\end{array}$ & $65(10.2)$ & $42(6.9)$ & $45(11.7)$ \\
$\quad$ Total energy, kcal/day & $2070(1690-2490)$ & $2060(1730-2450)$ & $2150(1730-2560)$ \\
$\quad$ Total fat intake, & $77(60-96)$ & $77(61-96)$ & $2110(1720-2560)$ \\
$\quad$ Fibre intake & $22(17-27)$ & $23(18-27)$ & $22(18-28)$ \\
Fruit and vegetable intake & $369(246-524)$ & $418(263-566)$ & $356(244-504)$ \\
$\quad$ Red meat intake & $48(25-77)$ & $48(26-76)$ & $56(34-84)$ \\
Processed meat intake & $25(13-41)$ & $23(12-42)$ & $364(250-524)$ \\
Alcohol intake & $9(1-23)$ & $8(1-21)$ & $54(31-82)$ \\
Hs-CRP, mg/l, median (IQR) & $3.1(1.3-5.7)$ & $2.2(1.1-4.7)$ & $26(13-47)$ \\
Cholesterol, mmol/L, median (IQR) & $6.3(5.5-7.1)$ & $6.4(5.6-7.2)$ & $2.3(1.0-4.4)$ \\
HDL, mmol/L, median (IQR) & $1.4(1.1-1.7)$ & $1.4(1.2-1.8)$ & $6.4(5.6-7.1)$ \\
LDL, mmol/L, median (IQR) & $4.2(3.5-4.8)$ & $4.2(3.5-4.9)$ & $1.4(1.2-1.7)$ \\
Glycer-AGEs, U/mL, median (IQR) & $6.8(5.3-8.3)$ & $7.0(5.5-8.7)$ & $4.2(3.4-4.8)$
\end{tabular}

NOTE: Cases and controls were matched on age (within 2.5 years), gender, administrative centre, hormone therapy and menopausal status (among women), fasting status, and date of blood collection (within 45 days)

Abbreviation: $S D$ = standard deviation; IQR = inter-quartile range; hs-CRP = high-sensitivity C-reactive protein; HDL = high-density lipoprotein; $L D L=$ low-density lipoprotein; Glycer-AGEs = glyceraldehyde-derived advanced glycation end-products 
Table 2: Circulating glycer-AGEs concentration and the risk of cancers of the colorectum, colon, and rectum

\begin{tabular}{|c|c|c|c|c|c|}
\hline \multirow[b]{3}{*}{ Type of Cancer } & \multicolumn{4}{|c|}{ Glycer-AGEs } & \multirow{3}{*}{$\mathrm{p}_{\text {trend }}$} \\
\hline & Q1 (reference) & Q2 & Q3 & Q4 & \\
\hline & OR & OR $(95 \% \mathrm{Cl})$ & OR $(95 \% \mathrm{Cl})$ & OR $(95 \% \mathrm{Cl})$ & \\
\hline \multicolumn{6}{|l|}{ Colorectum } \\
\hline Cut-point $[\mathrm{U} / \mathrm{mL}]^{\mathrm{a}}$ & $\leq 5.42$ & $>5.42$ and $\leq 7.03$ & $>7.03$ and $\leq 8.65$ & $>8.65$ & \\
\hline Mean (SD), median $[\mathrm{U} / \mathrm{mL}]$ & $4.3(0.9), 4.4$ & $6.3(0.5), 6.3$ & $7.8(0.4), 7.7$ & $10.5(1.7), 10.0$ & \\
\hline Number of cases/controls & $249 / 264$ & $301 / 264$ & $237 / 263$ & $266 / 263$ & \\
\hline Matching factors ${ }^{\mathrm{b}}$ & 1.00 & $1.19(0.94-1.50)$ & $0.95(0.74-1.23)$ & $1.07(0.81-1.42)$ & 0.95 \\
\hline Multivariate adjusted $^{c}$ & 1.00 & $1.18(0.92-1.51)$ & $0.94(0.72-1.24)$ & $1.10(0.82-1.49)$ & 0.87 \\
\hline \multicolumn{6}{|l|}{ Colon } \\
\hline Cut-point $[\mathrm{U} / \mathrm{mL}]^{\mathrm{a}}$ & $\leq 5.48$ & $>5.48$ and $\leq 6.99$ & $>6.99$ and $\leq 8.68$ & $>8.68$ & \\
\hline Mean (SD), median $[\mathrm{U} / \mathrm{mL}]$ & $\overline{4} .3(0.9), 4.5$ & $6.3(0.5), 6.2$ & $7.7(0.5), 7.7$ & $10.5(1.7), 10.0$ & \\
\hline Number of cases/controls & $174 / 166$ & $184 / 165$ & $157 / 164$ & $144 / 164$ & \\
\hline Matching factors ${ }^{b}$ & 1.00 & $1.03(0.77-1.37)$ & $0.88(0.64-1.20)$ & $0.78(0.55-1.11)$ & 0.12 \\
\hline Multivariate adjusted $^{c}$ & 1.00 & $1.04(0.76-1.42)$ & $0.86(0.61-1.22)$ & $0.83(0.57-1.22)$ & 0.25 \\
\hline \multicolumn{6}{|l|}{ Rectum } \\
\hline Cut-point $[\mathrm{U} / \mathrm{mL}]^{\mathrm{a}}$ & $\leq 5.36$ & $>5.36$ and $\leq 7.12$ & $>7.12$ and $\leq 8.61$ & $>8.61$ & \\
\hline Mean (SD), median [U/mL] & $4.2(1.0), 4.4$ & $6.3(0.5), \overline{6} .4$ & $7.8(0.4), 7.7$ & $10.3(1.7), 9.5$ & \\
\hline Number of cases/controls & $77 / 99$ & $115 / 100$ & $79 / 98$ & $125 / 99$ & \\
\hline Matching factors ${ }^{\mathrm{b}}$ & 1.00 & $1.49(0.99-2.23)$ & $1.14(0.73-1.80)$ & $1.90(1.17-3.10)$ & 0.03 \\
\hline Multivariate adjusted $^{c}$ & 1.00 & $1.47(0.96-2.27)$ & $1.11(0.68-1.80)$ & $1.90(1.14-3.19)$ & 0.04 \\
\hline
\end{tabular}

${ }^{\mathrm{a}}$ Based on control participants only

${ }^{b}$ Model based on matching factors (age, gender, administrative centre, time of the day at blood collection, fasting status, and menopausal status among women) only

${ }^{c}$ Model based on matching factors plus adjustments for smoking status/duration/intensity, body mass index, total physical activity, education level, diabetes status, total dietary energy consumption, and intakes of alcohol, red and processed meat, fibre, and fruits and vegetable

Abbreviation: Glycer-AGEs = glyceraldehyde-derived advanced glycation end-products; $\mathrm{Q}=$ quartile; $\mathrm{OR}=$ odds ratio; $\mathrm{Cl}=$ confidence interval 
Table 3: Odds ratios $(95 \% \mathrm{Cl})$ for colon and rectal cancer according to quartiles of circulating glycer-AGEs by alcohol intake status

\begin{tabular}{|c|c|c|c|c|c|}
\hline \multirow{3}{*}{$\begin{array}{c}\text { Sex specific categories of } \\
\text { dietary alcohol intake level (g/day) }\end{array}$} & \multicolumn{4}{|c|}{ Glycer-AGEs $^{a}$} & \multirow{3}{*}{$p_{\text {trend }}$} \\
\hline & Q1 & Q2 & Q3 & Q4 & \\
\hline & OR & OR $(95 \% \mathrm{Cl})$ & OR $(95 \% \mathrm{Cl})$ & OR $(95 \% \mathrm{Cl})$ & \\
\hline \multicolumn{6}{|l|}{ Colon } \\
\hline Number of cases/controls & $135 / 130$ & $145 / 137$ & $119 / 119$ & $104 / 124$ & \\
\hline Matching factors ${ }^{c}$ & 1.00 & $0.97(0.69-1.37)$ & $0.79(0.54-1.15)$ & $0.71(0.46-1.10)$ & 0.08 \\
\hline Multivariate adjusted $^{\mathrm{d}}$ & 1.00 & $1.01(0.69-1.47)$ & $0.79(0.52-1.20)$ & $0.82(0.51-1.33)$ & 0.29 \\
\hline \multicolumn{6}{|l|}{ High alcohol status (> median) ${ }^{b}$} \\
\hline Number of cases/controls & $39 / 36$ & $39 / 28$ & $38 / 45$ & $40 / 40$ & \\
\hline Matching factors ${ }^{c}$ & 1.00 & $1.48(0.68-3.20)$ & $0.92(0.40-2.07)$ & $1.11(0.47-2.58)$ & 0.95 \\
\hline Multivariate adjusted $^{d}$ & 1.00 & $1.10(0.46-2.61)$ & $0.78(0.31-1.95)$ & $1.17(0.46-3.03)$ & 0.95 \\
\hline \multicolumn{6}{|l|}{ Rectum } \\
\hline \multicolumn{6}{|l|}{ Low alcohol status $(\leq \text { median })^{\mathrm{b}}$} \\
\hline Number of cases/controls & $51 / 64$ & $70 / 57$ & $38 / 46$ & $57 / 63$ & \\
\hline Matching factors ${ }^{c}$ & 1.00 & $1.56(0.92-2.65)$ & $0.95(0.52-1.73)$ & $1.14(0.66-1.97)$ & 0.95 \\
\hline Multivariate adjusted $^{d}$ & 1.00 & $1.48(0.85-2.58)$ & $0.95(0.51-1.78)$ & $1.14(0.64-2.03)$ & 0.99 \\
\hline \multicolumn{6}{|l|}{ High alcohol status (> median) ${ }^{b}$} \\
\hline Matching factors ${ }^{c}$ & 1.00 & 1.39 (0.69-2.77) & $1.14(0.57-2.29)$ & $2.85(1.41-5.79)$ & $<0.01$ \\
\hline Multivariate adjusted $^{\mathrm{d}}$ & 1.00 & $1.41(0.69-2.88)$ & $1.07(0.52-2.19)$ & $2.70(1.29-5.62)$ & 0.01 \\
\hline
\end{tabular}

${ }^{a}$ Quartile cut-offs are same as in Table 2

${ }^{b}$ Alcohol consumption level was dichotomized based on the sex-specific median values of lifetime alcohol consumption among controls

${ }^{\mathrm{c}}$ Model adjusted for matching factors (age, gender, administrative centre, time of the day at blood collection, fasting status, and menopausal status among women) only

${ }^{\mathrm{d}}$ Model adjusted for matching factors plus smoking status/duration/intensity, body mass index, total physical activity, education level, diabetes status, total dietary energy consumption, red and processed meat, fibre, and fruits and vegetable

Abbreviation: Glycer-AGEs = glyceraldehyde-derived advanced glycation end-products; $\mathrm{Q}=$ quartile; $\mathrm{OR}=$ odds ratio; $\mathrm{Cl}=$ confidence interval 


\section{Cancer Epidemiology, Biomarkers \& Prevention}

\section{The Association between Glyceraldehyde-derived Advanced Glycation End-Products and Colorectal Cancer Risk}

So Yeon Kong, Masayoshi Takeuchi, Hideyuki Hyogo, et al.

Cancer Epidemiol Biomarkers Prev Published OnlineFirst September 24, 2015.

\begin{tabular}{|c|c|}
\hline Updated version & $\begin{array}{l}\text { Access the most recent version of this article at: } \\
\text { doi:10.1158/1055-9965.EPI-15-0422 }\end{array}$ \\
\hline Auth & $\begin{array}{l}\text { Author manuscripts have been peer reviewed and accepted for publication but have not yet been } \\
\text { edited. }\end{array}$ \\
\hline
\end{tabular}

E-mail alerts Sign up to receive free email-alerts related to this article or journal.

Reprints and To order reprints of this article or to subscribe to the journal, contact the AACR Publications Subscriptions Department at pubs@aacr.org.

Permissions To request permission to re-use all or part of this article, contact the AACR Publications Department at permissions@aacr.org. 